\title{
Foreword
}

\section{Correlations, connections, and physics in complex systems}

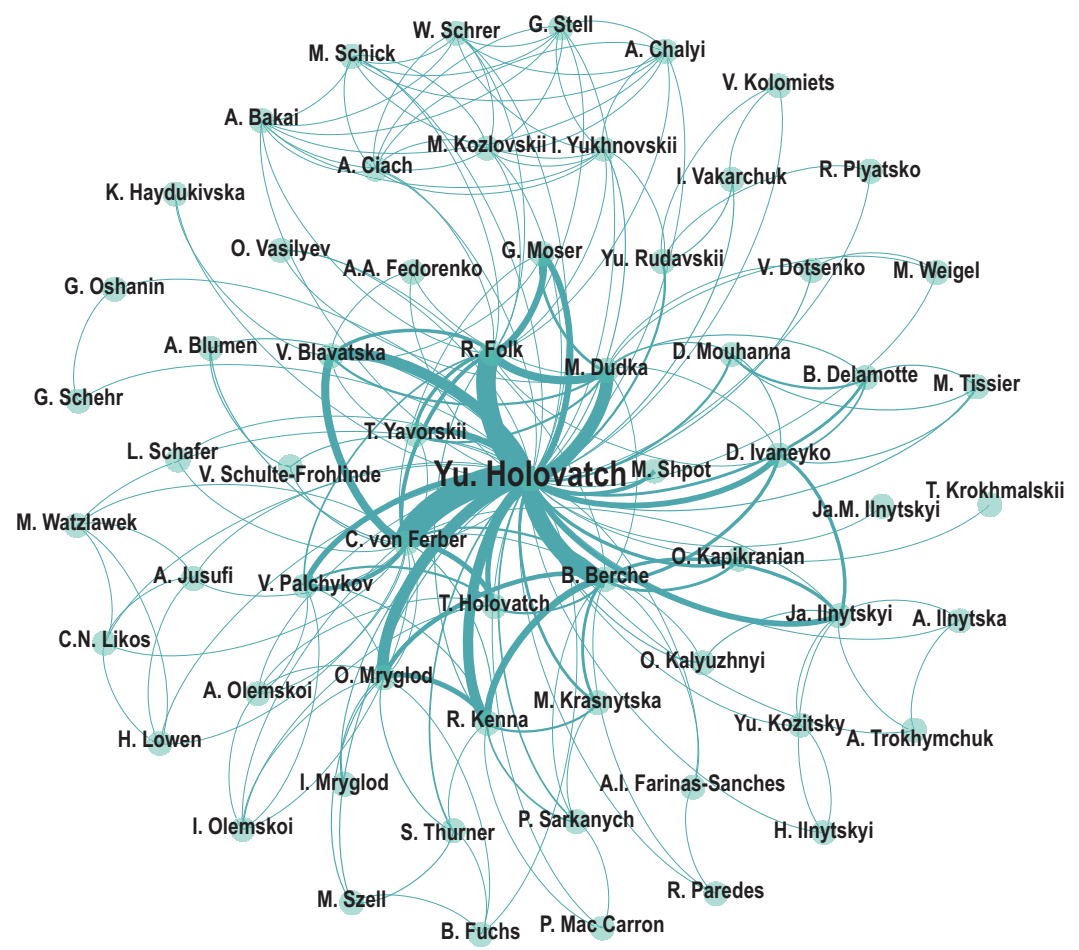

It is with great pleasure that we present, in this issue, a series of papers dedicated to the 60th birthday of Professor Yurij Holovatch. The broad spectrum of topics treated by the contributions reflect Yurij's comprehensive interests and his wide-ranging research. Thus, contributions range from papers on phasetransition theory to papers dealing with scientometric issues, from condensed matter theory to cosmological and astronomical problems.

Yurij's broad activities are not only reflected in the scope of his publications but also in his activities disseminating physics into other fields and initiating communications between scientists. For example, he founded the now-famous Ising lectures, which for the past two decades have been an important beacon for statistical physics in Europe. The associated review series Order Disorder and Criticality: Advanced Problems of Phase Transition Theory have been single-handedly edited by Yurij and published by World Scientific since 2004. By now, these contain 21 important and influential chapters describing the state of the art by 25 authorities in the field. In 2010 Yurij founded the Laboratory for Statistical Physics of Complex Systems at the Institute for Condensed Matter Physics in Lviv. There, he maintains regular activities with the members of his group, having seminars every week, even through modern communication tools when the majority of the members are abroad. Yurij co-founded the interdisciplinary seminar series Horizon of Science where problems at the cutting edge of modern science are discussed. A set of talks from these seminars were recently published (in Ukrainian). More recently, he initiated an exciting series of interdisciplinary workshops titled Physics Meets Humanities to demonstrate the applicability of the concepts and methods of science in social areas. 
Over his career, Yurij Holovatch has generated 165 papers in peer-reviewed journals. Through these, he has created a co-authorship network of scientists that we are proud to present in the figure. The majority of these scientific links appeared in the second half of Yurij's career. After Ukraine became independent in 1991, his activities increased considerably. This may be ascribed to Yurij's open disposition to take part in international programs and to create similar possibilities for young people.

Herein below we list a selection of Yurij's most important papers from which one can see the profound contributions he has made to different areas in physics [1-34]. One notices the evolution of his interests. After approximately 15 years working with classical topics in the physics of phase transitions and critical phenomena where he brought his formidable knowledge of the renormalization group to bear on problems such as random Ising models and continuous-symmetry models [1-5], he started to become interested in the applications of statistical physics in different areas.

Each of us has our own memories of the first scientific contact with Yurko and here we give short, personal recollections. R.F. first met Yurko at a conference organized near Uzhhorod, Ukraine, in the year 1994. At that time, both were working on phase transitions using renormalization group theory. Yurko was familiar with resummation methods and R.F. had the field theoretic functions for the transition into the superconducting phase to hand. Merging their experiences led to their first common publication [6] confirming the stability of a new "charged" fixed point. This nice beginning was then followed over the next years by considering more complicated magnetic systems involving dilution [9, 12, 13], anisotropies [10] and the combinations of both [15]. Not only did the topics become broader but also other colleagues joined the collaboration. More recently, and shortly before R.F. retired, dynamical properties near a phase transition have been considered. The dynamical model was a three-component antiferromagnet in three spatial dimensions in an external magnetic field, which contains several important subclasses, including model $\mathrm{F}$ for the condensation to the superfluid phase and model $\mathrm{C}$ for condensation to a magnetic phase including the coupling between the non-conserved alternating magnetization and a conserved quantity. The dynamics of an antiferromagnet have a variety of static and dynamic fixed points and the elaboration of all different cases lead to a series of papers ending in [26] which treats the most complicated case including reversible terms in dynamics.

B.B. first met Yurko during the 1995 MECO meeting in Austria, but they really became friends on the occasion of the 2000 meeting in France, where Yurko, speaking several languages fluently, was very helpful in solving some of the participants' problems. Besides Yurko's scientific work, an essential aspect of his friendly personality which makes him so valuable to his collaborators is a kind of skill in the manner of contact with others, and especially with young scientists with whom he is always open and curious for discussions, not only on science but also on life in general. B.B. witnessed this, in particular, in an unconventional context through his interactions with the participants - mostly students - of the Topical School held in Mochima, Venezuela in 2005, where Yurko gave a series of lectures on the renormalization-group approach to phase transitions [17]. Their scientific collaboration started with the study of the effect of disorder in the two-dimensional $X Y$ model [14]. B.B. then had the pleasure to co-advise, with Yurko, several PhD students on various topics: the role and mutual interaction of spinwaves and vacancies in two-dimensional topological phase transitions [18], public-transport networks [23, 28], spin models on complex networks [30]. To detail one of these topics only, it is worth mentioning the study of public-transport networks using the tools developed in the field of statistical physics of complex systems. Initiated in collaboration between Yurko and his long-established collaborator Christian von Ferber, this approach appeared to be very fruitful. In [19, 22], they offered different types of representations of the networks, the computation of network characteristic quantities and the relation between these quantities and features which are of importance for passengers. They have realized a study of major cities of the world in most of the continents. Although coming from a very different scientific community, and mostly published in physics journals, their contributions opened a new approach in the field and are very highly cited by the community of public transport studies. B.B. was involved in the continuation of this work, with comparison with random walks or percolation problems, resilience and robustness properties [23, 28]. B.B. is convinced that transmitting his knowledge to the next generation is an important aspect of Yurko's approach to his role. As he often saw him contact his students, Yurko is an open-minded and generous mentor.

R.K. first witnessed Yurko's energy and versatility at the remarkable Topical School in Venezuela in 2005 - the World Year of Physics. Although friendship was immediate, it took another 5 years, and 
Yurko's scientific vision, to co-author a paper. Since network science burst onto the scene around 20 years ago, Yurko was to the fore in adapting statistical physics to the new paradigm. An example of this was Yurko's spotting that R.K.'s work on logarithmic scaling relations could be applied to phase transitions on networks (rather than on regular lattices). R.K. readily accepted Yurko's invitation to join his highly successful collaboration and an appropriate team was assembled to investigate. In [24], new scaling relations for such exponents were developed and tested on a neat model with coupled order parameters on a scale-free network. R.K.'s collaboration with Yurko continued, and in [30], the Ising model on graphs of two different types were studied. A beautiful integral representation for the partition function on scalefree networks was found which recovers the complete graph in certain circumstances. The partition function zeros of the full range of models were investigated and, surprisingly, those in the complex field plane were found not to obey the celebrated Lee-Yang circle theorem. This important paper was selected for the Institute of Physics" "Publishers Pick" and the journal published an interview with its authors. In [32], the mean-field Potts model with additional non-interacting (invisible) states was considered. The model explains discrepancies between theoretical predictions and experimental observations of phase transitions in some systems where the symmetry is spontaneously broken. This insightful paper introduced a fully new mechanism for the emergence of the discontinuity characteristic in first-order phase transitions.

As a representative of Yurko's pupils, O.M. recalls one of her first personal conversations with him. In some sense it was already about complex networks, because Yurko was delighted about the artwork "Constructor" by Jacques Hnizdovsky. It was eventually adopted as a symbol for the Laboratory for Statistical Physics of Complex Systems. This small episode demonstrates some of the many features of Yurko's personality: his enthusiastic attitude to the new; his wonderful ability to see the hidden connections; his love for art; and his readiness to share his ideas. Thus, it was from complex network theory [19] that O.M. and Yurko started their joint journey. An attempt at a comprehensive analysis of a scientific journal based on bibliographic data can be considered as their first scientometric task. Along with the construction and analysis of different networks, the temporal properties of editorial processing within the journal were also studied [27]. Further work in the field of scientometrics includes the study of the evolution of scientific topics [31]; the analysis of downloads as an alternative way to measure the popularity of scientific papers and the comparison of quantitative and qualitative assessments of research groups, etc. Since science is frequently itself considered to be a very special kind of complex, social system, the research area expanded to involve sociophysical problems. In particular, an exciting joint venture was the temporal features of a social system within the virtual world, namely massive multiplayer online game PARDUS [29].

The above recollections illustrate how Yurko's interests brought him to transcend disciplinary confines as his interests evolved from condensed-matter physics to complexity research. Yurko's authoritative and broad knowledge enabled him to write a number of timely reviews. These include one of the first on complex networks which was very important in introducing new powerful theory, accompanied by new terminology, to a Ukrainian audience [19]. A more recent review aims to define, for once and for all, what precisely a complex system is [34]. This paper comes at a pivotal time in the theory of complex systems and is set to become a marker for the field in the years to come.

Yurko Holovatch is a true gentleman, whose science transcends scientific delineations as his personality transcends national and cultural borders. On behalf of his many appreciative colleagues and students, and of the wider scientific community, we wish Yurko every success for his future scientific projects and fruitful cooperations. We have no doubt that, as it has been in our cases, as the "Holovatch-network" continues to grow, new friendships will form. We look forward to these with immense gratitude.

Bertrand Berche (Université de Lorraine, Statistical Physics Group, Nancy, France)

Reinhard Folk (University Linz, Institute for Theoretical Physics, Linz, Austria)

Ralph Kenna (Applied Mathematics Research Centre, Coventry University, England)

Olesya Mryglod (Institute for Condensed Matter Physics of the National Academy of Sciences of Ukraine, Lviv, Ukraine) 


\section{References}

1. Vakarchuk I.O., Rudavskii Yu.K., Holovatch Yu.V., Analysis of Critical Properties of the $n$-Component Stanley Model on the Basis of the Approximate Renormalization Group Transformation, Phys. Many-Part. Syst., 1983, 4, 44-59 (in Russian).

2. Yukhnovskii I.R., Rudavskii Yu.K., Holovatch Yu.V., Analysis of Critical Behaviour of the $n$-Component Stanley Model in the Frames of Collective Variables Method, In: Thermodynamics of Irreversible Processes, Lopushanska A.I. (Ed.), Nauka, Moscow, 1987, 49-55 (in Russian).

3. Holovatch Yu., Shpot N., Critical Exponents of Random Ising-Like Systems in General Dimensions, J. Stat. Phys., 1992, 66, 867-883; doi 10.1007/BF01055706

4. Holovatch Yu., Phase Transition in Continuous Symmetry Model in General Dimensions: Fixed Dimension Renormalization Group Approach, Int. J. Mod. Phys. A, 1993, 8, 5329-5351; doi 10.1142/S0217751X93002113 [Saclay Preprint, Service de Physique Theorique, SPhT/123, 1992].

5. Holovatch Yu., Krokhmal's'kii T., Compilation of Two-Point and Four-Point Graphs in Field Theory in Non-Integer Dimensions, J. Math. Phys., 1994, 35, 3866-3880; doi 10.1063/1.530452

6. Folk R., Holovatch Yu., On the Critical Fluctuations in Superconductors, J. Phys. A, 1996, 29, 3409-3425; doi $10.1088 / 0305-4470 / 29 / 13 / 014$.

7. Von Ferber C., Holovatch Yu., Copolymer Networks and Stars: Scaling Exponents, Phys. Rev. E, 1997, 56, 63706386; doi 10.1103/PhysRevE.56.6370 [Preprint arXiv:cond-mat/9705278, 1997].

8. Von Ferber C., Holovatch Yu., Multifractality of Brownian Motion near Absorbing Polymers, Phys. Rev. E, 1999, 59, 6914-6923; doi 10.1103/PhysRevE.59.6914 [Preprint arXiv:cond-mat/9812119, 1998].

9. Folk R., Holovatch Yu., Yavors'kii T., Effective and Asymptotic Critical Exponents of Weakly Diluted Quenched Ising Model: 3d Approach Versus $\varepsilon^{1 / 2}$-Expansion, Phys. Rev. B, 2000, 61, 15114-15129; doi 10.1103/PhysRevB.61.15114 [Preprint arXiv:cond-mat/9909121] 2000].

10. Folk R., Holovatch Yu., Yavors'kii T., Pseudo- $\varepsilon$ Expansion of Six-Loop Renormalization Group Functions of an Anisotropic Cubic Model, Phys. Rev. B, 2000, 62, 12195-12200; doi 10.1103/PhysRevB.62.12195 [Preprint arXiv:cond-mat/0003216 2000], Erratum: Phys. Rev. B, 2001, 63, 189901(E); doi 10.1103/PhysRevB.63.189901

11. Blavats'ka V., von Ferber C., Holovatch Yu., Polymers in Long-Range-Correlated Disorder, Phys. Rev. E, 2001, 64, 041102; doi 10.1103/PhysRevE.64.041102 [Preprint arXiv:cond-mat/0107135, 2001].

12. Holovatch Yu., Blavats'ka V., Dudka M., von Ferber C., Folk R., Yavors'kii T., Weak Quenched Disorder and Criticality: Resummation of Asymptotic(?) Series, Int. J. Mod. Phys. B, 2002, 16, 4027-4079; doi 10.1142/S0217979202014760 [Preprint arXiv:cond-mat/0111158. 2002].

13. Folk R., Holovatch Yu., Yavors'kii T., Critical Exponents of a Three-Dimensional Weakly Diluted Quenched Ising Model, Usp. Fiz. Nauk, 2003, 173, 175; doi 10.3367/UFNr.0173.200302c.0175 (in Russian) [Phys. Usp., 2003, 46, 169; doi 10.1070/PU2003v046n02ABEH001077, Preprint arXiv:cond-mat/0106468, 2001].

14. Berche B., Fariňas-Sánches A.I., Holovatch Yu., Paredes R., Influence of Quenched Dilution on the Quasi-LongRange Ordered Phase of the 2d XY Model, Eur. Phys. J. B, 2003, 36, 91; doi 10.1140/epjb/e2003-00310-5 [Preprint arXiv:cond-mat/0309501 2003].

15. Dudka M., Folk R., Holovatch Yu., Critical Properties of Random Anisotropy Magnets, J. Magn. Magn. Mater., 2005, 294, 305-329; doi 10.1016/j.jmmm.2004.12.028 [Preprint arXiv:cond-mat/0406692 2004].

16. Blavats'ka V., von Ferber C., Holovatch Yu., Entropy-Induced Separation of Star Polymers in Porous Media, Phys. Rev. E, 2006, 74, 031801; doi 10.1103/PhysRevE.74.031801 [Preprint arXiv:cond-mat/0605503 2006].

17. Holovatch Yu., Introduction to Renormalization, Condens. Matter Phys., 2006, 9, 237-262; doi 10.5488/CMP.9.2.237

18. Kapikranian O., Berche B., Holovatch Yu., The 2D XY Model on a Finite Lattice with Structural Disorder: QuasiLong-Range Ordering under Realistic Conditions, Eur. Phys. J. B, 2007, 56, 93-105; doi 10.1140/epjb/e2007-00095-5 [Preprint arXiv:cond-mat/0612147] 2006].

19. Holovatch Yu., von Ferber C., Olemskoi O., Holovatch T., Mryglod O., Olemskoi I., Palchykov V., Complex Networks, J. Phys. Stud., 2006, 10, 247-291.

20. Holovatch Yu., Palchykov V., Mykyta the Fox and Networks of Language, J. Phys. Stud., 2007, 11, 22-33 [Preprint arXiv:0705.1298 2007].

21. Ivaneyko D., Berche B., Holovatch Yu., Ilnytskyi J., On the Universality Class of the $3 \mathrm{~d}$ Ising Model with Long-Range-Correlated Disorder, Physica A, 2008, 387, 4497-4512; doi 10.1016/j.physa.2008.03.034 [Preprint arXiv:cond-mat/0611568 2009].

22. Von Ferber C., Holovatch T., Holovatch Yu., Palchykov V., Public Transport Networks: Empirical Analysis and Modeling, Eur. Phys. J. B, 2009, 68, 261-275; doi 10.1140/epjb/e2009-00090-x [Preprint arXiv:0803.3514 2008].

23. Berche B., von Ferber C., Holovatch T., Holovatch Yu., Resilience of Public Transport Networks against Attacks, Eur. Phys. J. B, 2009, 71, 125-137; doi 10.1140/epjb/e2009-00291-3 
24. Palchykov V., von Ferber C., Folk R., Holovatch Yu., Kenna R., Critical Phenomena on Scale-Free Networks: Logarithmic Corrections and Scaling Functions, Phys. Rev. E, 2010, 82, 011145; doi 10.1103/PhysRevE.82.011145 [Preprint arXiv:1004.0097 2010].

25. Delamotte B., Dudka M., Holovatch Yu., Mouhanna D., About the Relevance of the Fixed Dimension Perturbative Approach to Frustrated Magnets in Two and Three Dimensions, Phys. Rev. B, 2010, 82, 104432; doi 10.1103/PhysRevB.82.104432 [Preprint arXiv:1009.1492 2010].

26. Folk R., Holovatch Yu., Moser G., Field Theory of Bicritical and Tetracritical Points. IV. Critical Dynamics Including Reversible Terms, Phys. Rev. E, 2012, 85, 021143; doi 10.1103/PhysRevE.85.021143

27. Mryglod O., Holovatch Yu., Mryglod I., Editorial Process in Scientific Journals: Analysis and Modeling, Scientometrics, 2012, 91, 101-112; doi 10.1007/s11192-011-0536-1[Preprint arXiv:1109.6211]2011].

28. Von Ferber C., Berche B., Holovatch T., Holovatch Yu., A Tale of Two Cities. Vulnerabilities of the London and Paris Transit Networks, J. Transp. Secur., 2012, 5, 199-216; doi 10.1007/s12198-012-0092-9 [Preprint arXiv:1206.2599. 2012].

29. Mryglod O., Fuchs B., Szell M., Holovatch Yu., Thurner S., Interevent Time Distributions of Human MultiLevel Activity in a Virtual World, Physica A, 2015, 419, 681-690; doi 10.1016/j.physa.2014.09.056 [Preprint arXiv:1407.2006 2014].

30. Krasnytska M., Berche B., Holovatch Yu., Kenna R., Partition Function Zeros for the Ising Model on Complete Graphs and on Annealed Scale-Free Networks, J. Phys. A, 2016, 49, 135001; doi 10.1088/1751-8113/49/13/135001 [Preprint arXiv:1510.00534 2015].

31. Mryglod O., Holovatch Yu., Kenna R., Berche B., Quantifying the Evolution of a Scientific Topic: Reaction of the Academic Community to the Chornobyl Disaster, Scientometrics, 2016, 106, 1151-1166; doi 10.1007/s11192-015-1820-2 [Preprint arXiv:1511.05797] 2015].

32. Krasnytska M., Sarkanych P., Berche B., Holovatch Yu., Kenna R., Marginal Dimensions of the Potts Model with Invisible States, J. Phys. A, 2016, 49, 255001; doi 10.1088/1751-8113/49/25/255001 [Preprint arXiv:1512.03635 2015].

33. Kalyuzhnyi O., Ilnytskyi Ja., Holovatch Yu., von Ferber C., Universal Shape Characteristics for the Mesoscopic Polymer Chain via Dissipative Particle Dynamics, J. Phys.: Condens. Matter, 2016, 28, 505101; doi 10.1088/0953-8984/28/50/505101] [Preprint arXiv:1606.08666. 2016].

34. Holovatch Yu., Kenna R., Thurner S., Complex Systems: Physics beyond Physics, Eur. J. Phys., 2017, 38, 023002; doi 10.1088/1361-6404/aa5a87 [Preprint arXiv:1610.01002 2016]. 